\title{
Review: \\ Microwave-Promoted Organic Synthesis
}

\author{
Andrew Loftin and Douglas Armstrong \\ Department of Physical Sciences \\ Olivet Nazarene University \\ Bourbonnais, Illinois 60914 USA
}

Received: December 1, 2009

Accepted: December 20, 2009

\begin{abstract}
As of the last twenty years a new wave in organic chemistry has started to change the way people think about putting energy into a reaction mixture. Microwave-promoted organic synthesis is a fast, efficient method of heating a system in order to achieve the completion of a desired reaction. There are many different types of reactions that have been attempted using this method, and still many more to be tested. Microwave-promoted organic synthesis can help to produce high yields of products in a short time, while reducing side reactions and making workups easier. This paper discusses the many benefits of microwave-promoted organic synthesis and gives various examples.
\end{abstract}

\section{INTRODUCTION}

Ever since the very earliest experiments in the science of chemistry, some type of energy has often been used to drive chemical reactions. One of the earliest examples of this was heat from a Bunsen burner. Later other sources of heat were used such as heating mantels, hot water baths, and oil baths. Eventually, other forms of energy were found to drive reactions, including electricity, light and even sound [1]. Then around 1986 Geyde and Giguere/Majetich published some papers describing the use of microwaves to heat organic reactants to produce products [2]. This enormous breakthrough has had many chemists looking for new and innovative ways to use microwave-promoted organic synthesis, including Suzuki reactions, nucleophilic aromatic substitutions, DielsAlder reactions, etc.

\section{HOW DOES IT WORK?}

Like light, microwaves are a form of electromagnetic radiation. Different substances may interact differently with microwaves, but only three ways are possible, as follows [3]. Microwaves can be absorbed, reflected or transmitted. Very few materials do only one of these three. Most substances do a little of each of the three. Microwaves interact with polar and ionic species most easily [3]. The substances attempt to align themselves with the electric current being created by the microwaves [1]. To determine how easily microwaves heat a substance, chemists use a number called a loss tangent. The equation for this is:

$$
\tan \delta=\varepsilon^{\prime} / \mathcal{\varepsilon}
$$

where $\varepsilon^{\prime \prime}$ is the dielectric loss, or the ability of the substance to convert electromagnetic radiation into heat, and $\varepsilon^{\prime}$ is the dielectric constant or the ability of the polar molecule to align itself with the electric field [2]. Essentially, the higher the value for the loss tangent, the better the molecule absorbs microwave energy.

Therefore, for syntheses with microwave radiation, this information is important when choosing solvents and reactants. If neither the solvent nor any reactant absorbs microwave energy, they are transparent to it; the reaction mixture will not be heated [2]. However, if either the solvent or a reactant (or both) absorbs microwave energy, heating of the reaction mixture will occur relatively easily. The interesting thing is that it only takes one, either the reactants or the solvent, to absorb 
the microwave energy. This is because as one heats up, it causes everything it is touching to heat up as well, thus heating the entire reaction mixture by convection [2]. One large advantage this has over traditional heating methods is, with microwave radiation, the energy is being applied directly to the system [2]. Traditionally, heat was applied through hot water baths, heating mantles, sand baths, or some other means that requires that the container holding the substances be heated first in order for their contents to be heated. In fact with microwave chemistry, it is preferred that the reaction container not be heated. This is because it would require some of the microwaves to interact with the substance the container is made from, when they could simply pass through and interact with the reactants or solvent directly [2].

\section{EXAMPLES OF MICROWAVE- PROMOTED REACTIONS}

One example of a reaction that is being carried out using microwave synthesis is the Suzuki coupling reaction. A Suzuki reaction is a palladium-catalyzed crosscoupling of aryl halides with boronic acids [2]. Suzuki coupling is used for its simple and efficient creation of biaryls [4]. These biaryls are often found in pharmaceuticals, herbicides, and natural products [4]. In a 2003 study, Leadbeater compared the microwave-promoted Suzuki reaction with that of a conventional heating method. The expectation was that the microwavepromoted reaction would take significantly less time, as is the case with most other reactions. However, this was not the case. The conventional method actually yielded about $94 \%$ in five minutes, compared to $91 \%$ yield in the same amount of time from the microwave-promoted reaction carried out in the same way [4]. Leadbeater's determination was that this seemed to be probably an exception to the normal Suzuki reaction and he even states that this was the fastest Suzuki coupling he had ever heard of [4]. He also states that the microwave version of his experiment was much more easily controlled, because the microwave system allows control of temperature, pressure, and reaction time with very high precision [4].

$$
\text { In a later paper Leadbeater }
$$

discussed another benefit of this Suzuki microwave procedure. Using the work of Pivonka and Empfield, he constructed a device that measures in situ Raman spectroscopy [5]. This allowed for the reaction to be monitored throughout the entire reaction time without stopping the reaction to examine the products using another spectrophotometer [5].

One of the more difficult types of reactions to complete is a nucleophilic aromatic substitution. This is due mainly to the fact that an aromatic ring is a rich source of electrons. Nucleophiles are reluctant to react with aromatic rings due to the high electron density of the rings. Of course it is possible to use electron-withdrawing groups to pull some electron density away from the ring. However this does not always ensure success. Many times reactions of this nature require high temperatures and long reaction times [2]. In 2004 the Van der Eycken group worked with pyrido-fused heterocycles, and discovered that with water as a solvent they could complete the substitution of o-flouro-benzaldehyde with pyrrolidine at $130^{\circ} \mathrm{C}$ for 3 minutes [2]. This gives sufficient hope for the possibility that other aromatic nucleophilic substitutions may eventually be carried out in a microwave setting.

One of the best known types of organic reactions is the Diels-Alder reaction. A simple Diels-Alder reaction can take more than 20 minutes while heating at a given reaction temperature. However, Chen and colleagues [6], studying Diels-Alder reactions using microwaves, discovered that by using a Lewis acid as a catalyst they could carry out a reaction within the period of about a minute [2,6].

Since many microwave instruments have multiple holders for reactions vials, this equates to many reactions that can be carried out in a very short time period. This time advantage is so significant that many Diels-Alder type reactions are being carried out using microwaves. One of these is the one-pot asymmetric synthesis of biaryl compounds using Diels-Alder/Retro-DielsAlder cascade reactions [7]. These results are important because they selectively allow production of biaryl compounds which contain chirality resulting, not from a tetrahedral center, but from a chiral axis. These axially chiral biaryls play a role in 
governing properties of bioactive molecules [7].

\section{CONCLUSION}

Microwave chemistry has started to change the face of chemical reactions as we know it. Microwave chemistry is the "wave of the future". For reactions that will work in a microwave setting there are many advantages. The reaction temperature, pressure, and time can be very carefully and automatically controlled. With microwave synthesis there is no longer a need to constantly tinker with knobs on a heating mantle. In fact almost the entire reaction can be automated. Also, as seen by Leadbeater's developments $[4,5]$ with the Suzuki reactions, there may be ways to use the microwave setting to measure quantities of substances while the reaction is going on. This allows for the progress of the reaction to be determined without stopping it. Also, in many cases reactions may take place at lower temperatures. This benefit often results in higher yields, fewer occurrences and lower volumes of "side products," and easier, more efficient work-ups. However, perhaps the biggest benefit is the overall speed with which the reactions take place. Some reactions that normally take a long while may be completed in only a few minutes.

Another benefit is that microwave synthesis may be more environmentally friendly. Many of the reactions mentioned in this article were carried out using water as the solvent. Water is the most harmless of all substances found in a chemistry lab, and the more it can be utilized, the better.

Microwave-promoted chemistry is a quickly growing field, and may one day supplant traditional heating methods for many types of reactions. It is very important that more research be done to determine what reactions would do well in a microwave setting, and to develop procedures for these reactions.

\section{REFERENCES}

1. Suslick, Kenneth S. "Sonochemistry." Access Science. Web. 30 May 2009. <http://www.accessscience.com.proxy.ol ivet.edu/content.aspx? searchStr=sonoc hemistry\&id=637005>.

2. Dallinger, Doris, and C. Oliver Kappe. "Microwave-Assisted Synthesis in Water as Solvent." Chemical Reviews 107(6) (2007), pp. 2563-591. American Chemical Society. Web. 28 May 2009. <http://pubs.acs.org/?cookieSet=1>.

3. Varma, Rajender S. "Microwave organic synthesis." Access Science. Web. 6 June 2009. <http://www.accessscience.com.proxy.ol ivet.edu/content.aspx? searchStr=Micro wave+Organic+Synthesis\&id $=$ YB02057 $0>$.

4. Leadbeater, Nicholas E., and Maria Marco. "Rapid and Amenable Suzuki Coupling Reaction in Water Using Microwave and Conventional Heating." The Journal of Organic Chemistry 68(3) (2003), pp. 888-92. American Chemical Society. Web. 18 May 2009. <http://pubs.acs.org/?cookieSet=1>.

5. Leadbeater, Nicholas E., and Rebecca J. Smith. "Real-Time Monitoring of Microwave-Promoted Suzuki Coupling Reactions Using in Situ Raman Spectroscopy." Organic Letters 20(8) (2006), pp. 4589-591. American Chemical Society. Web. 28 May 2009. <http://pubs.acs.org/?cookieSet=1>.

6. Chen, I. H., Young, J. N., Yu, S. J. Tetrahedron (2004).

7. Liu, Yongxiang, Kui Lu, Mingji Dai, Wanqing Wu, Jiahua Chen, Junmin Quan, and Zhen Yang. "An Efficient One-Pot Asymmetric Synthesis of Biaryl Compounds." Organic Letters (2007), pp. 805-808. American Chemical Society. Web. 30 May 2009. $<$ http://pubs.acs.org/?cookieSet=1>. 